\title{
Subconjunctival Hemorrhage in a Child with the Blue Rubber Bleb Nevus Syndrome on Treatment with Oral Propranolol
}

\author{
Karen De Loecker ${ }^{a} \quad$ Veerle Labarque $^{b} \quad$ Hilde Seynaeve $^{c} \quad$ Ingele Casteels $^{a}$ \\ aDepartment of Ophthalmology, University Hospitals Leuven, Leuven, Belgium; \\ ${ }^{b}$ Department Pediatric Hematology, University Hospitals Leuven, Leuven, Belgium; \\ 'Department of Ophthalmology, AZ Delta, Roeselare, Belgium
}

\section{Keywords}

Blue rubber bleb nevus syndrome $\cdot$ Subconjunctival hemorrhage $\cdot$ Topical $\beta$-blocker · Case report

\begin{abstract}
Blue rubber bleb nevus syndrome (BRBNS) is a rare syndrome characterized by venous malformations of mostly skin and gastrointestinal tract. Patients present with multiple venous malformations in various organs including liver, spleen, heart, eye, and central nervous system. Few ophthalmological cases have been published in literature and at present, there are no clear guidelines on the treatment of eye hemorrhages associated with the BRBNS. We report a 3-year-old boy with the BRBNS who developed a spontaneous progressive enlarging subconjunctival hemorrhage in the left eye despite being treated with oral propranolol. The subconjunctival hemorrhage was caused by an underlying conjunctival vascular malformation. With topical treatment with timolol maleate $0.5 \%$ once a day in the affected eye, the lesion regressed completely after 4 months. This child represents the first case of the BRBNS associated with a subconjunctival progressive bleeding necessitating topical treatment despite oral propranolol effectively controlling the cutaneous lesions. We recommend ophthalmic screening of patients with BRBNS in early childhood. For patients with BRBNS-related subconjunctival vascular lesions with subsequent hemorrhage, treatment with a topical $\beta$-blocker may be an efficient and harmless treatment option.
\end{abstract}




\section{Introduction}

Blue rubber bleb nevus syndrome (BRBNS) usually presents at birth or shortly thereafter. Most cases are sporadic; however, autosomal dominant inheritance has been reported with a locus found on chromosome $9 p[1,2]$. Patients with BRBNS have characteristic vascular malformations, most commonly found on the skin and in the gastrointestinal tract. Less frequent localizations of the lesions are in the brain, joint, liver, spleen, kidney, and eye. The majority of patients suffer from intestinal bleedings leading to anemia. Here we report on a 3-year-old boy with the BRBNS who presented with a subconjunctival hemorrhage caused by an underlying vascular malformation, treated with a topical $\beta$-blocker.

\section{Case Report/Case Presentation}

A 3-year-old boy presented at our emergency department with a spontaneous progressive enlarging subconjunctival hemorrhage. The boy was the first-born baby to healthy nonconsanguineous parents. He was born after a normal pregnancy and delivery with a birthweight of $3.230 \mathrm{~kg}$. Immediately after birth, several vascular lesions were noted on his feet (shown in Fig. 1). Over the next months, more cutaneous lesions appeared all over the body, as well as submucosal lesions in the mouth. An MRI of the forearm was performed and revealed dispersed vascular lesions without the presence of an enchondroma leading to the clinical diagnosis of BRBNS. Biopsies of the lesions on the left thigh and right flank were taken at the age of 3 months, and the diagnosis of BRBNS was confirmed. Treatment with oral propranolol was started, and the dose $(2 \mathrm{mg} / \mathrm{kg} /$ day) was increased over time according to the weight. The majority of the submucosal lesions in the oral cavity was fluctuating with some cutaneous lesions fading away but also new cutaneous lesions emerging. Overall, the number and size of lesions clearly decreased over the years. In addition, the boy never presented with iron deficiency anemia nor clinical symptoms suggestive for gastrointestinal tract involvement. At the age of 3 years, he presented at the emergency department with a small subconjunctival hemorrhage on the left eye. Mother mentioned the appearance of a small red punctiform lesion 10 days before the hemorrhage emerged, suggesting a new vascular lesion in a child with the BRBNS. History revealed no recent trauma to the eye and the child did not have hypertension. The peripheral blood count including platelet count was within normal limits.

Fig. 1. After birth, several vascular lesions were noted on his feet.

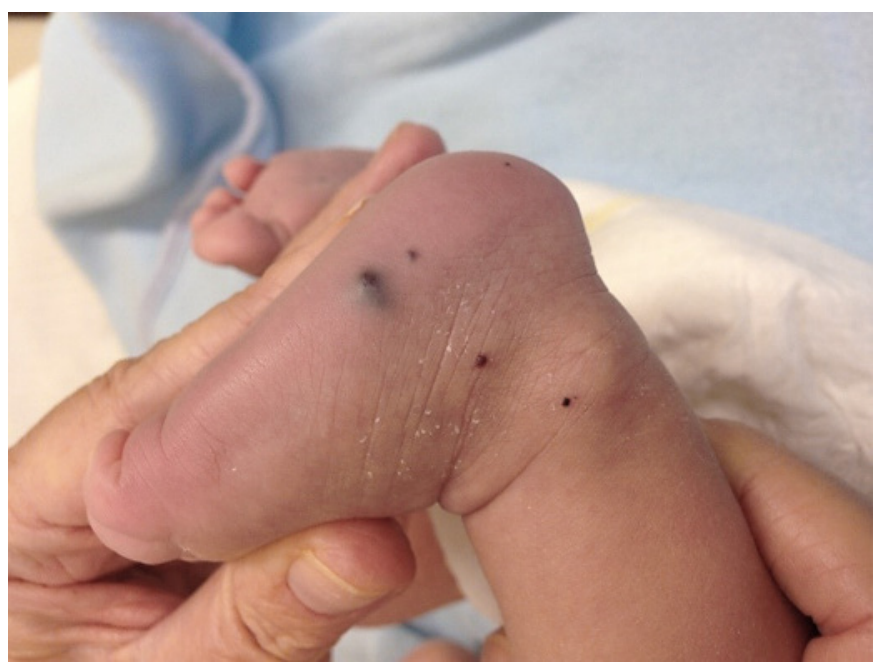


No additional treatment was started. Three days later, however, he was urgently referred because of progressive subconjunctival hemorrhage (shown in Fig. 2a). Topical treatment with timolol maleate $0.5 \%$ eye drop once a day was started. After 2 weeks, progressive resorption of the hemorrhage was seen (shown in Fig. 2b). After 1 and a half month, the bleeding subsided completely (shown in Fig. 2c) with after 4 months only a slight irregularity of the conjunctiva (shown in Fig. 2d). Topical treatment with timolol maleate $0.5 \%$ was stopped after 6 months and to date, after 1 year of follow-up, there has been no relapse.

\section{Discussion/Conclusion}

In 1860, Gascoyen [3] reported the association of cutaneous nevi, intestinal lesions and gastrointestinal bleeding. The term "blue rubber bleb naevus syndrome" was initiated in 1958 by Bean [4] due to the color and consistency of the cutaneous vascular malformations having the feeling and appearance of rubber nipples. Bean described 3 types of vascular lesions: one with large disfiguring cavernous angiomas; the second as a compressible blood sac, like a blue rubber nipple covered by milk-white skin; and finally, irregular blue marked punctate spots. BRBNS is characterized by venous malformations of the skin, gastrointestinal tract, and less commonly of other organs including central nervous system, eye, joints, muscles, liver, spleen, lungs, kidney, heart, parotic gland, and bladder. The disease is very rare; only 200 cases have been reported [5]. Most cases are sporadic. However, autosomal dominant inheritance has been reported with a locus on chromosome $9 \mathrm{p}$, characterized by somatic double (cis) mutations in the TEK gene (TIE2) [6]. This is an endothelial cell tyrosine kinase receptor for angiopoietins [1, 2, 7-9]. The peculiar bluish cutaneous lesions are mostly present at birth or early childhood and vary in size from 1 to $5 \mathrm{~cm}$ and can increase in size and number over time, while visceral involvement tends to present in early adulthood. Yet, the severity and clinical course of the cutaneous lesions are not correlated with the severity
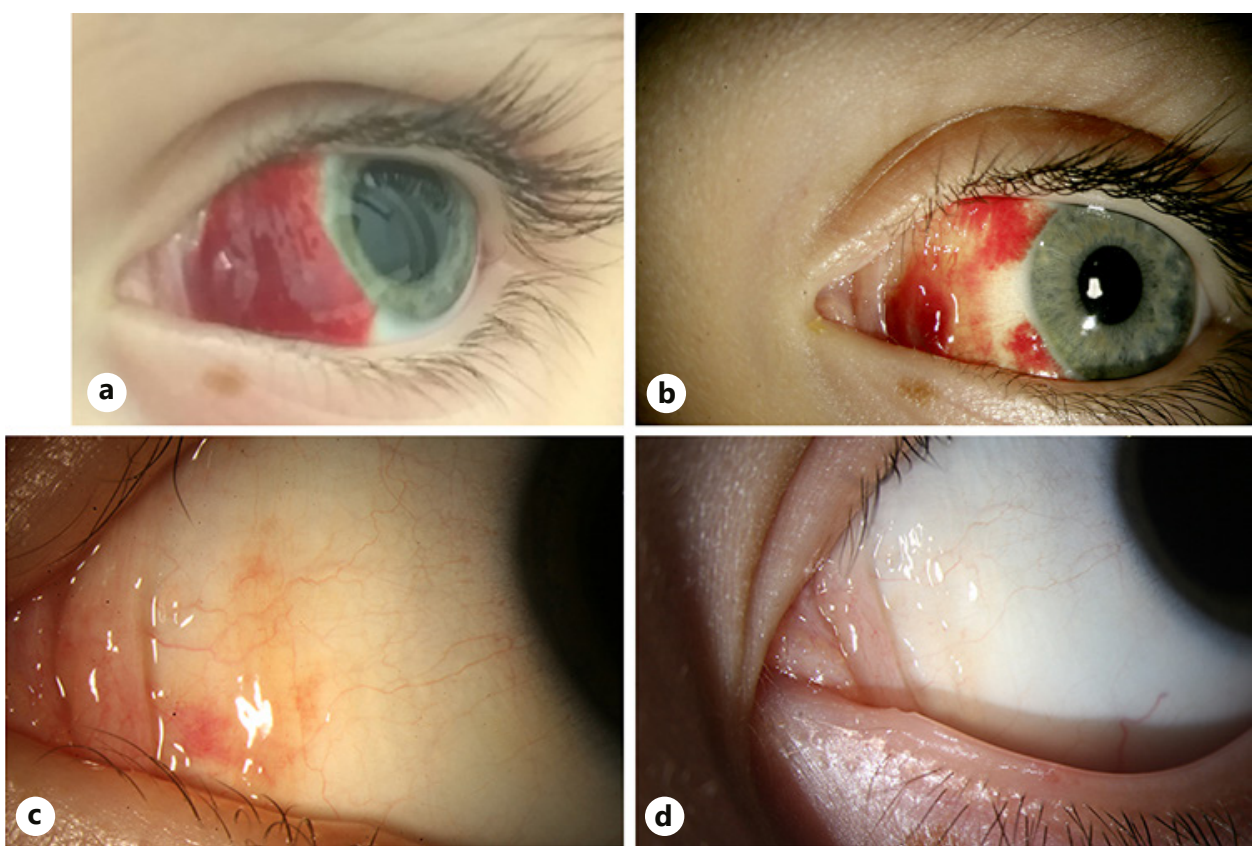

Fig. 2. a-d At presentation, progressive enlarging hemorrhage (a) with regression of hemorrhage when treated with topical timolol maleate $0.5 \%$ eye drop $1 \times$ /day after 2 weeks (b), 6 weeks (c), and 18 weeks (d). 
of the internal lesions. Ocular involvement is rare but may involve the orbit, iris, ciliary body and processes, conjunctiva, eyelid, choroidea, and macula. This can lead to complications such as diplopia, optic nerve compression, hyphema with secondary glaucoma, and vitreous or retinal hemorrhage. Only a few ophthalmic cases have been reported. The first report is by Burke et al. [10], reporting on 2 cases: one newborn with an iris lesion in the right eye who was also blind due to bilateral occipital lesions, and another baby with fundus lesions that regressed by 28 months.

In 1981, Crompton and Taylor [11] published another case of a 2-day-old male baby with the clinical and histological diagnosis of the BRBNS. In addition to several scattered nodular lesions on the skin, the ophthalmological examination showed a bulbar conjunctival hemangioma in the lefteye and a subconjunctival hemorrhage near the temporallimbus. Furthermore, there was a small hemangioma in the supratemporal quadrant of the left iris which had bled into the anterior stroma. In the left macula, there was a raised hemorrhagic lesion, suggestive for an arteriovenous malformation. All the lesions regressed spontaneously [11]. In 1997, Chen et al. [12] reported the first childhood-onset BRBNS with cutaneous and ocular lesions in a 9-year-old girl. On CT scan of the brain she was found to have bilateral orbital intraconal lesions and a left vertex nodule. During the ophthalmological examination, a hemangioma was visualized on the lid margin and tarsal conjunctiva of the left eye. On ophthalmoscopy, a submacular choroidal lesion was diagnosed. Follow-up after 6 months did not show any change [12]. To date, few adult cases have been published. In 2005, Shannon and Auld [13] reported a 83-year-old woman with rapid visual loss due to undiagnosed BRBNS and cortical blindness caused by a hemorrhage in both occipital lobes. On MRI of the brain, multiple cerebral and cerebellar cavernomas were visualized. Shams et al. [14] described a 57-year-old woman, known with BRBNS, with an acute proptosis due to a thrombosis within an orbital varix of the superior ophthalmic vein. This resolved spontaneously within a few days. Finally, Petek and Jones [15] published a case of a 59-year-old man, who was diagnosed with a familial type of BRBNS and developed a dural arteriovenous fistula in his orbit. He presented with visual loss in the right eye and elevated intraocular pressure, proptosis and chronic pain in the left eye. Multiple endovascular embolization procedures resolved all his ocular symptoms. In this paper, Petek and Jones [15] also give an overview of the potential treatment options. Surgical treatment of chronic ophthalmic manifestations has been successful in most of the patients. Endovascular embolization has been used to alleviate symptoms in patients with dural arteriovenous fistulas; multiple procedures may be required. In addition, surgical excision can be successful in patients with other venous malformations in the orbit [15-17]. On the other hand, systemic therapy has not yet been successful in treating ophthalmic involvement of BRBNS. Nevertheless, sirolimus has achieved promising outcomes in other features of the disease. Recent publications have suggested that sirolimus may be beneficial in the treatment of gastrointestinal bleedings and anemia. Therefore, this agent can be considered for short-term treatment of ophthalmic malformations, but cautious long-term use is needed as it may expose patients to side effects, including hypertension, immunosuppression, renal failure, anemia, and hyperlipidemia [18-21].

We here report a 3-year-old boy with the diagnosis of BRBNS and cutaneous lesions who was treated with oral propranolol. Despite the good response and control on the cutaneous lesions, our patient developed a subconjunctival hemorrhage upon a subconjunctival vascular malformation. At first no additional treatment was initiated, but progressive and acute bleeding was seen after 3 days. Topical treatment with timolol maleate $0.5 \%$ was started and resulted in a progressive regression of the subconjunctival hemorrhage and hemangioma.

We report the first child with BRBNS and a subconjunctival hemangioma and progressive hemorrhage necessitating topical treatment despite oral propranolol effectively controlling

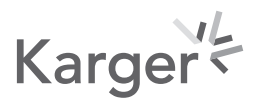


the cutaneous lesions. Although we cannot prove that the clearance of the bleeding is the result of the topical treatment, we conclude that for patients with this BRBNS-related subconjunctival lesion, treatment with a topical $\beta$-blocker may be an efficient and harmless treatment option.

\section{Statement of Ethics}

The parents of the patient gave oral and written consent to publish the data. The report does not include personal information that could identify the patient directly or indirectly. The study protocol was approved by the institute's committee on human research. This study was approved by the Ethics Committee Research University Hospitals Leuven and the reporting and writing are all in compliance with the Declaration of Helsinki.

\section{Conflict of Interest Statement}

The authors have no conflicts of interest to declare.

\section{Funding Sources}

No funding was received for this research or publication.

\section{Author Contributions}

Karen De Loecker together with Hilde Seynaeve collected the data, designed, drafted, and finalized the article. She also searched the literature on PubMed by choosing the following MeSH terms: blue rubber bleb nevus syndrome, therapy, and eye. Ingele Casteels was responsible for the medical treatment of the eye problem and has seen the patient at all intervals. Veerle Labarque is responsible for the medical treatment of all other BRBNS associated problems in this patient. Ingele Casteels, Veerle Labarque, and Hilde Seynaeve critically reviewed the several drafts and all authors approved the final version of the manuscript and are accountable for all aspects of the work. All authors attest that they meet the current ICMJE criteria for authorship.

\section{References}

1 Jin XL, Wang ZH, Xiao XB, Huang LS, Zhao XY. Blue rubber bleb nevus syndrome: a case report and literature review. World J Gastroenterol. 2014;20(45):17254-9.

2 Liu Q, Chen YP, Li YM. Blue rubber bleb nevus syndrome: a report of one case associated with recurrent epistaxis. Chin Med J. 2007;120(8):731-3.

3 Gascoyen M. Case of naevus involving the parotid gland and causing death from suffocation. Naevi of the viscera. Trans Pathol Soc. 1860;11:267.

4 Bean W. Vascular spiders and related lesions of the skin. Springfield, IL: Charles C Thomas; 1958. p. 178-85.

5 Baigrie D, An IC. Blue rubber bleb nevus syndrome. Statpearls. 2019.

6 Galione CJ, Pasyk KA, Boon LM, Lennon F, Johnson DW, Helmbold EA, et al. A gene for familial venous malformation maps to chromosome 9p in a second. Hum J Med Genet. 1995;32(3):197-9.

7 Soblet J, Kangas J, Nätynki M, Mendola A, Helaers R, Uebelhoer M, et al. Blue rubber bleb nevus syndrome is caused by somatic TEK (TIE2) mutations. J Invest Dermatol. 2017;137:207-16.

\section{Karger's}


8 Zahedi MJ, Darvish Moghadam S, Seyed Mirzaei SM, Dehghani M, Shafiei Pour S, Rasti A. Blue rubber bleb nevus syndrome as a rare cause of iron deficiency anemia: a case report and review of literature. Middle East J Dig Dis. 2013;5(4):235-9.

9 Jin XL, Wang ZH, Xiao XB, Huang LS, Zhao XY. Blue rubber bleb nevus syndrome: a case report and literature review. World J Gastroenterol. 2014;20(45):17254-9.

10 Burke EC, Winkelmann RK, Strickland MK. Disseminated hemangiomatosis. The newborn with central nervous system involvement. Am J Dis Child. 1964;108(4):418-24.

11 Crompton JL, Taylor D. Ocular lesions in the blue rubber bleb naevus syndrome. Br J Ophthalmol. 1981;65(2): 133-7.

12 Chen PP, Weishaar PD, Murray TG. Blue rubber bleb nevus syndrome. J Pediatr Ophthalmol Strabismus. 1997; 34(5):321-3.

13 Shannon J, Auld J. Blue rubber bleb naevus syndrome associated with cortical blindness. Australas J Dermatol. 2005;46(3):192-5.

14 Shams PN, Cugati S, Wells T, Huilgol S, Selva D. Orbital varix thrombosis and review of orbital vascular anomalies in blue rubber bleb nevus syndrome. Ophthal Plast Reconstr Surg. 2015;31(4):e82-6.

15 Petek B, Jones RL. The management of ophthalmic involvement in blue rubber bleb nevus syndrome. GMS Ophthalmol Cases. 2014;4:Doc04-4.

16 Arena M,Viridis M, Morandi E, Viaggi P, Pisani A, Opocher E, et al. Blue rubber bleb nevus syndrome: combined surgical and endoscopic treatment. Endoscopy. 2015;47(Suppl 1 UCTN):E372-3.

17 Chang EL, Rubin PA. Bilateral multifocal hemangiomas of the orbit in the blue rubber bleb nevus syndrome. Ophthalmology. 2002;109(3):537-41.

18 Yuksekkaya H, Ozbek O, Keser M, Toy H. Blue rubber bleb nevus syndrome: successful treatment with sirolimus. Pediatrics. 2012;129(4):e1080-4.

19 Fox VL. New therapies for vascular anomalies of the gastrointestinal tract. Minerva Pediatr. 2018;70(3): 303-7.

20 Isoldi S, Belsha D, Yeop I, Uc A, Zevit N, Mamula P, et al. Diagnosis and management of children with blue rubber bleb nevus syndrome: a multi-center case series. Dig Liver Dis. 2019;51(11):1537-46.

21 Gildener-Leapman JR, Rosenberg JB, Barmettler A. Proptosis reduction using sirolimus in a child with an orbital vascular malformation and blue rubber bleb nevus syndrome. Ophthal Plast Reconstr Surg. 2017; 33(3S Suppl 1):S143. 